\title{
Plastic intermittency during cyclic loading: From dislocation patterning to microcrack initiation
}

\author{
J. Weiss, ${ }^{1, *}$ W. Ben Rhouma, ${ }^{2}$ S. Deschanel, ${ }^{2}$ and L. Truskinovsky ${ }^{3}$ \\ ${ }^{1}$ Université Grenoble Alpes, CNRS, ISTerre, 38000 Grenoble, France \\ ${ }^{2}$ Université de Lyon, MATEIS, CNRS UMR 5510, INSA de Lyon, 69621, Villeurbanne, France \\ ${ }^{3}$ PMMH, CNRS UMR 7636, ESPCI ParisTech, 10 Rue Vauquelin, 75005 Paris, France
}

(Received 26 July 2018; revised manuscript received 3 January 2019; published 25 February 2019)

\begin{abstract}
In metallic materials subjected to cyclic loading, strain hardening as well as fatigue crack initiation have been linked for a long time with the evolution of dislocation patterns and structures. In particular, the development of low-energy dislocation configurations such as persistent slip bands (PSBs) is considered as a precursor to crack initiation. However, the associated scenarios have been elaborated mainly from postmortem observations capturing only static pictures of dislocation patterns, while the dynamics of the problem has been somewhat overlooked. Here we analyze collective dislocation dynamics during cycling loading of aluminum using acoustic emission (AE). A strong link is revealed between dislocation patterning, cyclic hardening/softening, and the intermittency of plasticity: Plastic intermittency and dislocation avalanches rapidly decay during the initial hardening stage, in conjunction with the reduction of an internal length scale characterizing the dislocation structure. However, in nonannealed samples, a transient softening stage ensues, associated with a brutal reorganization of this structure. These initial stages of cyclic deformation illustrate the competition between two phenomena: collective dislocation dynamics, governed by long-ranged elastic interactions among dislocations, and the emergence of a self-organizing network controlled by short-range interactions and progressively inhibiting collective effects. Later on, the emergence of PSBs is accompanied by a reincrease of the AE intermittent activity. We propose that the associated AE bursts may be the signature of collective and coordinated dislocation motions along PSBs leading to the formation of incipient microcracks.
\end{abstract}

DOI: 10.1103/PhysRevMaterials.3.023603

\section{INTRODUCTION}

When subjected to cyclic mechanical loading, structural components can fail unexpectedly after hundreds to thousands of cycles. Rather remarkably, such failure occurs under stress levels well below the failure stress observed under monotonic loading. Fatigue failures are of primary importance in various industrial contexts, including transportation industries [1], aeronautics [2], or more recently microelectronics [3]. It has been estimated that more than half of mechanical ruptures during service are due to fatigue, which induces considerable economical costs $[4,5]$.

In metals, steels and alloys for which the plastic yield stress is reached at each cycle, strain hardening as well as fatigue crack initiation have been linked with the evolution of dislocation patterns and formation of cell structures during cyclic loading [6,7]. In particular, the development of lowenergy dislocation configurations such as persistent slip bands (PSBs) is associated with plastic strain localization, which serve as precursors to crack initiation [5,8]. Consequently, the evolution of dislocation patterning during fatigue has been extensively studied, and is now well characterized, at least for pure metals [9].

Most of these observations, however, were performed postmortem [e.g., from transmission electron microscopy (TEM)], hence capturing only static pictures of dislocation patterns

\footnotetext{
*jerome.weiss@univ-grenoble-alpes.fr
}

after a given number of loading cycles. In contrast, the dynamics of the problem has not been studied as thoroughly. More than 30 years ago, Laird and coworkers reported, directly from high-speed recording of the stress-strain hysteresis loops, the existence of transient mechanical instabilities during the cyclic loading of pure fcc metallic single crystals that they called "strain avalanches" [10-12]. These authors interpreted these events as collective and coordinated dislocation motions within PSBs and argued that such plastic instabilities might be associated with fatigue crack initiation. However, it was later understood that these instabilities could be largely machineinduced, providing only a highly distorted view of true plastic avalanches [13]. As a result, this topic was largely abandoned.

On the other hand, the problem of the intermittency of plastic deformation and collective dislocation dynamics, in a general sense, has been revived over the last two decades [14-17]. In classical plasticity theory, dislocation-mediated plastic flow is generally assumed to be smooth, with small and uncorrelated strain fluctuations averaged out and thus undetectable at large $(\geqslant \mathrm{mm})$ scales. This remained in contradiction with the fact that an intermittent character of plasticity in metals, with large fluctuations of the strain rates associated with dislocation avalanches, had been observed a long time ago, especially at the early stages of deformation in the microplastic regime of hcp metals [18-20]. Over the last decades, acoustic emission (AE) measurements performed on hcp materials (ice [14,21] and metals [22,23]), under monotonic loading, linked the intermittency with collective dislocation dynamics beyond the microplastic regime, and 
revealed the ubiquity of scale-free (power-law distributed) dislocation avalanches [15] with space [24] and time [25] clustering.

These two apparently conflicting pictures of crystalline plasticity were recently reconciled [26]: The small number of easy slip planes in hcp crystals favors a collective dislocation dynamics ruled by long-range elastic interactions, as well as kinematic hardening. The resulting collective effects manifesting themselves through large (possibly systemspanning) avalanches that are the signature of wild plasticity. These avalanches generate well-defined transient AE bursts, the so-called "discrete AE". On the reverse, the multislip character of plastic deformation in fcc materials favors shortrange interactions between dislocations such as the formation of locks and junctions that give rise to stable, low energy configurations $[27,28]$. These configurations represent strong barriers to dislocation motion and lead to complex threedimensional (3D) dislocation entanglements manifested by isotropic (forest) hardening [27]. The concomitant build-up of dislocation microstructures (walls, cells, ...) reduces the dislocation mean-free path [29] and therefore strongly frustrates collective dislocation dynamics and avalanches, leading to a mild plasticity compatible with the classical picture of smooth plastic flow. The AE signature of such mild plasticity is a slowly evolving background noise, the so-called "continuous AE" (see Refs. [26,30,31], and below). Note that the shortrange interactions mentioned above have to be distinguished from the double cross-slip of screw dislocations, which might be an efficient mechanism to by-pass obstacles, and is expected to play a significant role on plastic intermittency [32], including in hcp materials such as ice [33].

However, as it was shown in Ref. [26], even in this mild regime, where most of plastic deformation occurs through small uncorrelated dislocation motions, rare but potentially very large (relative to the characteristic wavelength of the dislocation microstructural pattern) cascades of unlocking events can take place, leading to brutal rearrangements of the dislocation substructures [26,34]. This argues for the existence of crossover regimes where mild (small and uncorrelated) and wild (correlated, power-law distributed) fluctuations can coexist. To characterize such a crossover, it is convenient to define a parameter measuring the degree of wildness $W$. A natural choice for such a measure is the fraction of plastic deformation accommodated through dislocation avalanches [26].

A more recent study has shown that the wild-to-mild transition is controlled, to the first order, by a ratio of length scales, $R=L / l$, with $L$ the external system size and $l$ an internal length scale [35]. The fact that $W$ decreases with $R$ explains how fcc and bcc pure metals characterized by smooth plastic flow at bulk scales may still exhibit power-law distributed strain bursts at $\mu \mathrm{m}$ to sub- $\mu \mathrm{m}$ scales (see, e.g., Refs. [36,37]). The internal length scale $l$ can be extrinsically set through alloying, allowing one to counteract the effect of the external size and thus tame intermittency of plastic deformation at small scales [35].

In pure metals, however, one expects $l$ to intrinsically emerge from short-range dislocation interactions and serve as a characteristic of a dislocation microstructure. In this context, $l$ can be identified as a typical scale of the dislocation pattern which, according to the "similitude principle" [38-40], should be inversely proportional to the shear stress $\tau, l \sim G b / \tau$, where $G$ is the shear modulus, and $b$ is the Burgers's vector. Combining this with the Taylor's relation describing strain hardening, $\tau \sim G b \sqrt{\rho}$ where $\rho$ is the (forest) dislocation density, we obtain $l \sim 1 / \sqrt{\rho}[39,41]$, which defines the boundary between short- and long-ranged (elastic) interactions between dislocations [40]. From these considerations, one can expect the wildness of plastic deformation $W$ : (i) to increase with decreasing initial dislocation density $\rho_{i}$; and (ii) to decrease as the material strain-hardens and the forest density $\rho$ increases.

As we have already mentioned, in fcc metals, cyclic deformation produces particularly well-defined low-energy dislocation structures such as walls, cells, and PSBs [6], which are behind cyclic strain hardening and ultimately induce fatigue crack initiation. Note that even if fractal dislocation patterns have been observed for copper subjected to monotonic tension [42], the microstructures generated under cyclic loading are generally characterized by a well-defined internal length scale $l$, the similitude principle applies [43], and one can try to relate the behavior of the degree of wildness $W$ in cyclic loading with the much better documented stress-strain response.

With this perspective in view, we pose in this paper the following questions:

(1) Do dislocation avalanches exist during the whole development of fatigue in fcc metals or plastic intermittency vanishes after only few loading cycles as a result of the build-up of stable, low-energy dislocation substructures?

(2) Do the anticipated relations between $W$, strainhardening, and the dislocation patterning scale $l$ hold under cyclic loading?

(3) Is there a link between plastic avalanches, PSBs, and fatigue crack initiation as originally proposed by Laird and coworkers?

To answer these questions we performed a thorough analysis of acoustic emission during cyclic deformation (fatigue test) of a typical fcc material, pure aluminum. Our conclusions vis a vis the questions $1-3$ are presented at the end of the paper.

\section{EXPERIMENTS}

We used an hydraulic machine, designed to minimize mechanical noise [31], to perform at room temperature a series of cyclic strain-controlled tension-compression tests on pure $(99.95 \%)$ aluminum polycrystals $\left(R_{\varepsilon}=\frac{\varepsilon_{\min }}{\varepsilon_{\max }}=-1 ; \Delta \varepsilon=\right.$ $\varepsilon_{\max }-\varepsilon_{\min }=0.95 \%$, corresponding to a plastic strain amplitude $\Delta \varepsilon_{p} \approx 0.85 \%$; loading frequency of $0.1 \mathrm{~Hz}$ ). The polycrystals were chosen to have large grain sizes $\left(L_{g} \sim 5-10\right.$ $\mathrm{mm}$ ), which ensures that grain boundaries do not affect the degree of wildness $W$, as $L_{g} \gg l$. Few samples were annealed at $250{ }^{\circ} \mathrm{C}$ for $2 \mathrm{~h}$ before testing in order to reduce the initial dislocation density $\rho_{i}$.

Acoustic emission was monitored using a PCI2 Mistras data acquisition system of Euro Physical Acoustics S.A. and piezoelectric sensors, with a preamplification of $60 \mathrm{~dB}$ and a $50 \mathrm{kHz}-1.2 \mathrm{MHz}$ frequency bandwidth. The raw AE signal was sampled by the PCI card at a frequency of $5 \mathrm{MHz}$. An example is shown in Fig. 1. This raw signal was used by the acquisition system to detect AE bursts over a predefined amplitude threshold of $34 \mathrm{~dB}$, and to determine their individual 


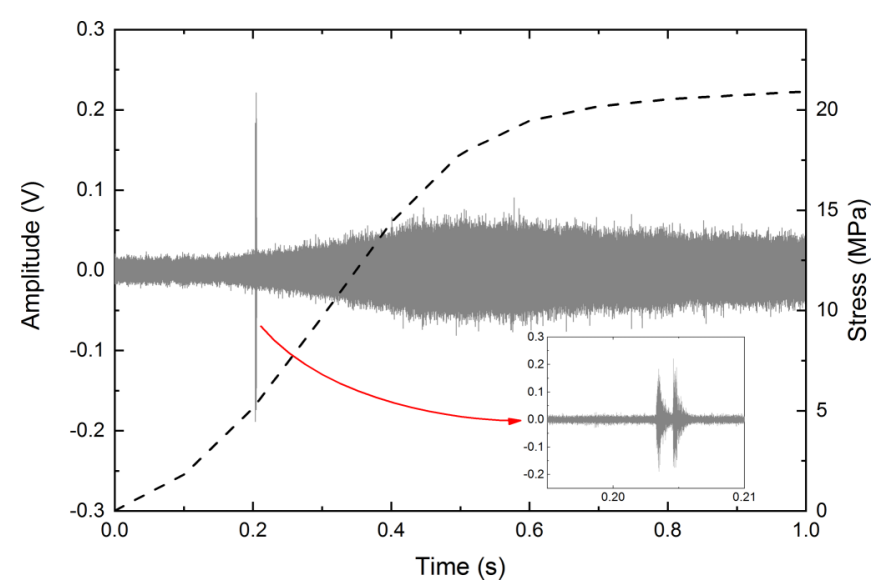

FIG. 1. The raw AE signal recorded during the onset of loading (cycle 1) for a cyclic strain-controlled tension-compression $\left(R_{\varepsilon}=\right.$ $\left.\frac{\varepsilon_{\min }}{\varepsilon_{\max }}=-1 ; \Delta \varepsilon=\varepsilon_{\max }-\varepsilon_{\min }=0.95 \%\right)$ test on a nonannealed polycrystalline $\mathrm{Al}$ sample (grey curve). The black dashed line represents the applied stress. Two well defined AE bursts, illustrating the discrete acoustic emission, are visible around $0.2 \mathrm{~s}$ (see inset for a zoom). The envelope of the signal, illustrating the continuous AE, initially increases, is maximum around plastic yield, and then slowly decays.

characteristics (arrival time, energy $E$, duration $\Delta t, \ldots$ ). The energy of an AE burst $i$ is given by the squared amplitude integrated over the burst duration, $E_{i}=\int_{\Delta t_{i}} A(t)^{2} d t$, while the burst duration is determined using predefined time constants (peak definition time $=300 \mu \mathrm{s}$; hit definition time $=600 \mu \mathrm{s}$; hit lockout time $=1 \mathrm{~ms}$ ). This standard protocol has been fully detailed elsewhere [44]. This raw signal is also used to calculate the AE power $\frac{d E}{d t}$. However, this power was not recorded at such a high frequency during all the tests, instead sampled continuously throughout the tests at a sampling frequency of $100 \mathrm{~Hz}$. This means that each sampled value of the AE power was obtained from an averaging of the squared $5 \mathrm{MHz}$ raw signal over a time window of $10 \mathrm{~ms}$,

$$
\frac{d E}{d t}=\frac{\int_{10 m s} A(t)^{2} d t}{10 m s} .
$$

Note that this AE power encompasses both continuous and discrete $\mathrm{AE}$ (if any).

In the material under study, the only sources of AE are dislocation depinning, microcrack nucleation, and abrupt crack advances, which all have to be distinguished. We have recently reported the detection of acoustic emissions specific to stage II fatigue incremental crack growth [44]. These acoustic multiplets are characterized by nearly identical waveforms repeatedly triggered over many successive loading cycles at almost the same stress and originating from a single location. From these unambiguous signatures of fatigue crack growth, we were able to identify the onset of stage II for each fatigue test. In what follows we focus only on the deformation stages preceding such crack growth. Consequently, the AE data presented below can be linked exclusively to dislocation rearrangements and microcrack nucleation along primary slip planes on stage I of the fatigue life of a sample.

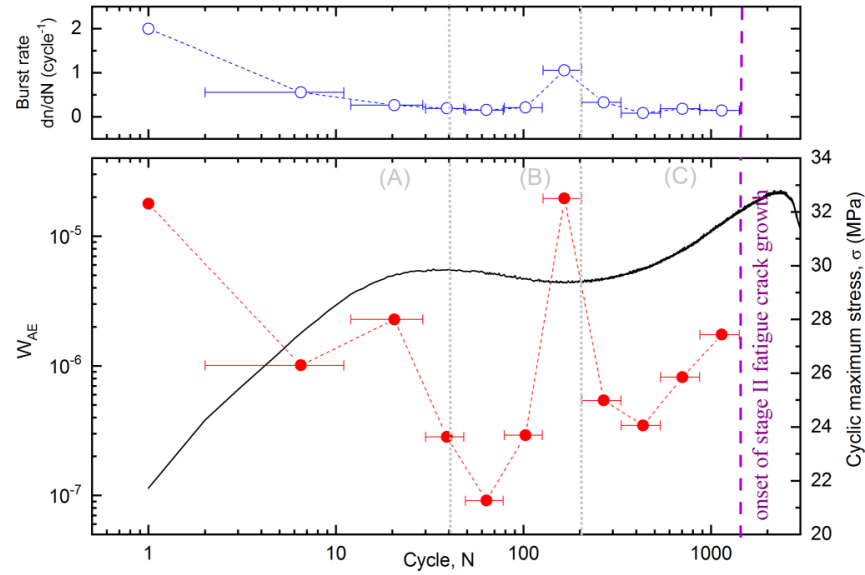

FIG. 2. Cyclic strain-controlled tension-compression $\left(R_{\varepsilon}=\right.$ $\left.\frac{\varepsilon_{\min }}{\varepsilon_{\max }}=-1 ; \Delta \varepsilon=\varepsilon_{\max }-\varepsilon_{\min }=0.95 \%\right)$ test on a nonannealed polycrystalline Al sample. The cyclic stress response (csr-curve) is shown as a black line on the bottom panel. The AE burst rate (number of discrete AE bursts per cycle) is shown on the top panel (blue open circles). The wildness proxy $W_{A E}$ is shown on the bottom panel (red closed circles). (A), (B), and (C) refer to the initial hardening, softening, and secondary hardening stages, respectively (see text for details). The vertical purple line indicates the onset of stage II fatigue crack growth detected from AE multiplets [44].

\section{CYCLIC DEFORMATION STAGES AND DISLOCATION PATTERNS}

The observed cyclic stress response (csr-curve) in a typical test on a nonannealed sample is shown in Fig. 2; it is in close agreement with earlier experiments [45,46]. To associate different stages of cyclic behavior with dislocation patterning, we relied on: (i) scanning electron microscopy (SEM) observations of the surface of a sample during a test performed under the same loading conditions but interrupted at various stages of fatigue life; (ii) previously published TEM observations for pure aluminum samples subjected to similar loading protocols ( $\Delta \varepsilon_{p}$ between 0.48 and $0.64 \%$ ) [45].

An initial, rapid hardening stage (A) (see Fig. 2), lasting $\sim 10-20$ cycles for $\Delta \varepsilon=0.95 \%$, is singled out by the development of dislocation entanglements leading to an irregular cell structure [45]. At this stage, the only detectable structures at the surface are slip lines on few grains, in a single-slip configuration [Fig. 3(a)].

A softening stage (B), lasting $\sim 100$ cycles, shows a considerable reorganization of the initial random dislocation pattern into a regular structure and straight dislocation walls [45]. Transient softening can be explained by this microstructural rearrangement, which is also characterized by the onset of the activation of additional slip systems, as double-slip configurations were observed within few grains [Fig. 3(b)].

Finally, a secondary hardening stage (C), which can last thousands of cycles and does not show any stress saturation, is associated with the development of a well-defined, regular wall and ladder structure [45]. At this stage the first signs of slip irreversibility and crack initiation along PSBs appear at the surface, see Fig. 3(c).

As the cyclic deformation continues, crack propagation becomes visible perpendicular to the loading axis, indicating 

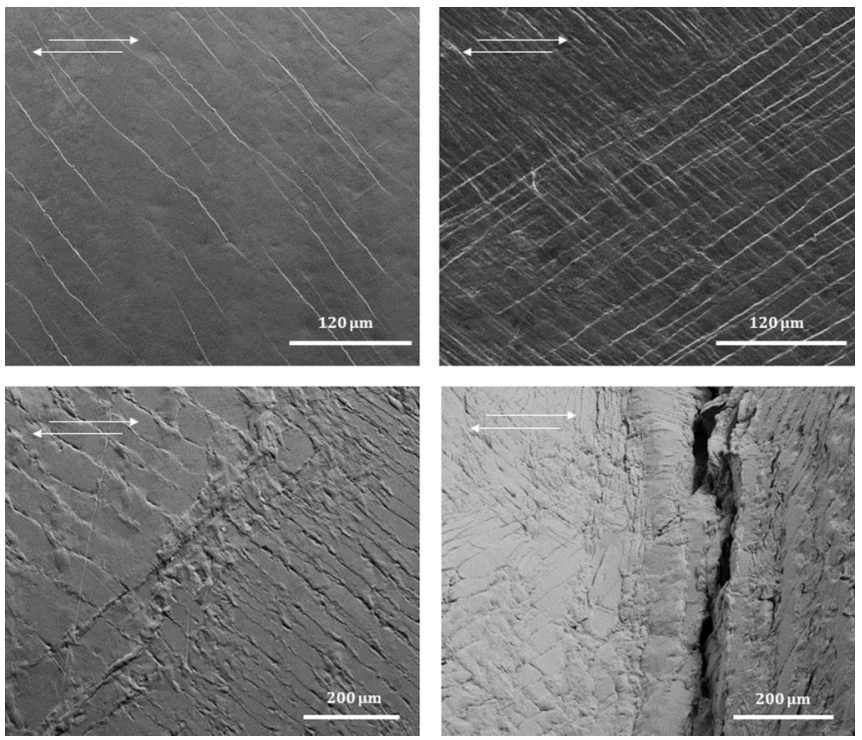

FIG. 3. SEM observations of the surface of a sample during a test $\left(R_{\varepsilon}=\frac{\varepsilon_{\min }}{\varepsilon_{\max }}=-1 ; \Delta \varepsilon=\varepsilon_{\max }-\varepsilon_{\min }=0.95 \%\right)$ interrupted at various stages of cyclic deformation: (a) 10 Cycles; (b) 100 cycles; (c) 1100 cycles, and (d) 2100 cycles. See Sec. III of the text for details. The white arrows indicate the tension-compression loading axis.

stage II of fatigue cracking [Fig. 3(d)]. This stage, as well as the final softening stage corresponding to extensive damage and final failure, will not be analyzed in this paper. Note that the features newly observed at a given stage of deformation (e.g., PSBs and crack initiation for stage C) were absent at the previous stages, on any grain.

\section{PLASTIC INTERMITTENCY}

In these experiments we identified two types of AE. Continuous AE, representing a noise-like signal (see Fig. 1), was always present during cyclic deformation. We interpret this signal as a signature of plastic deformation resulting from the cumulative effect of numerous, small, and uncorrelated (in space and in time) dislocation rearrangements. They are activated within independent dislocation "units" (cells, channels, ...), and are characterized by a size distribution with a well-defined scale [30,31]. In our classification, this is a signature of the mild plasticity. In this case, where many independent AE sources are assumed to overlap, the AE power scales as $\frac{d E}{d t} \sim b\langle S\rangle \frac{d \varepsilon_{p}}{d t}$, where $\langle S\rangle$ is an average sweeping area for these small dislocation motions [31,47], i.e., $\langle S\rangle^{1 / 2}$ represents a dislocation mean-free path, i.e., $\langle S\rangle^{1 / 2} \approx l$. Such correspondence between the continuous AE power $\frac{d E}{d t}$ and the plastic strain-rate $\frac{d \varepsilon_{p}}{d t}$ has been previously validated for monotonic [48] and cyclic loading [26] of Al, and is shown in Fig. 4.

Considering that the forest dislocation density increases during the work-hardening stage, and so the mean free path decreases, we expect, for a strain-rate controlled monotonic test, a very small AE power during the elastic stage, a maximum of power near plastic yield when $\langle S\rangle$ is maximum, and a slow decrease during the work-hardening stage when

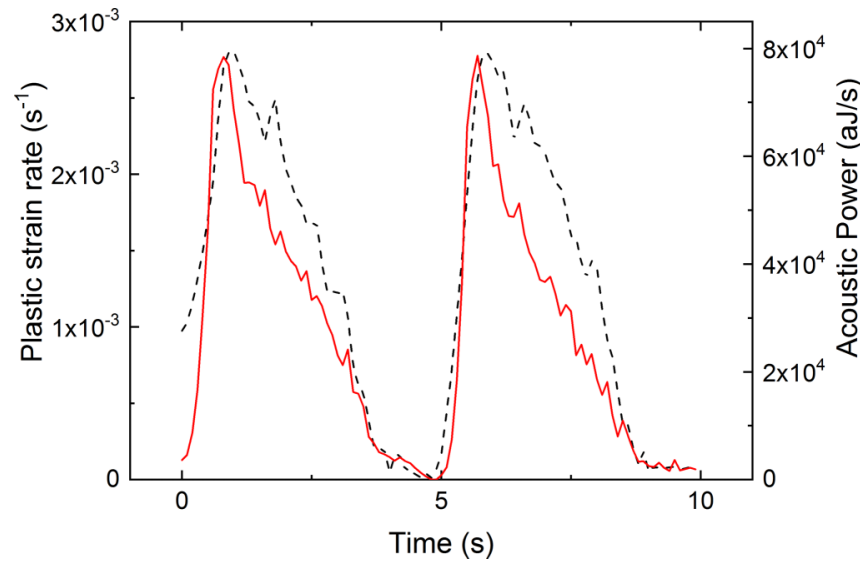

FIG. 4. Comparison between the AE power recorded during a loading cycle $\left(\mathrm{n}^{\circ} 329\right)$ of a test $\left(R_{\varepsilon}=\frac{\varepsilon_{\min }}{\varepsilon_{\max }}=-1 ; \Delta \varepsilon=\varepsilon_{\max }-\right.$ $\left.\varepsilon_{\min }=0.95 \%\right)$ performed on a nonannealed polycrystalline $\mathrm{Al}$ sample (red solid line), and the macroscopic plastic strain-rate measured by extensometry (dashed black line). The instrumental/environmental background noise has been removed from the recorded $\mathrm{AE}$ power.

$\langle S\rangle$ decreases but $\frac{d \varepsilon_{p}}{d t}$ remains roughly constant. This was observed previously [26], and is consistent with the envelope of the raw signal of Fig. 1. For the cyclic loading tests described here, this continuous AE source model can explain the decrease of the AE power peak value, recorded at plastic yield, with the increasing number of cycles during the early part of the tests, when cyclic strain hardening is important, and so the mean free path decreases [see Fig. 5(a)].

We also observed individual AE bursts added to this continuous background (see, e.g., Fig. 1). For their identification we used the thresholding technique mentioned above. The bursts represent the "discrete" AE and are the signature of intermittent dislocation avalanches $[21,23]$ that we associated with wild plasticity [26]. In this case, the maximum amplitude

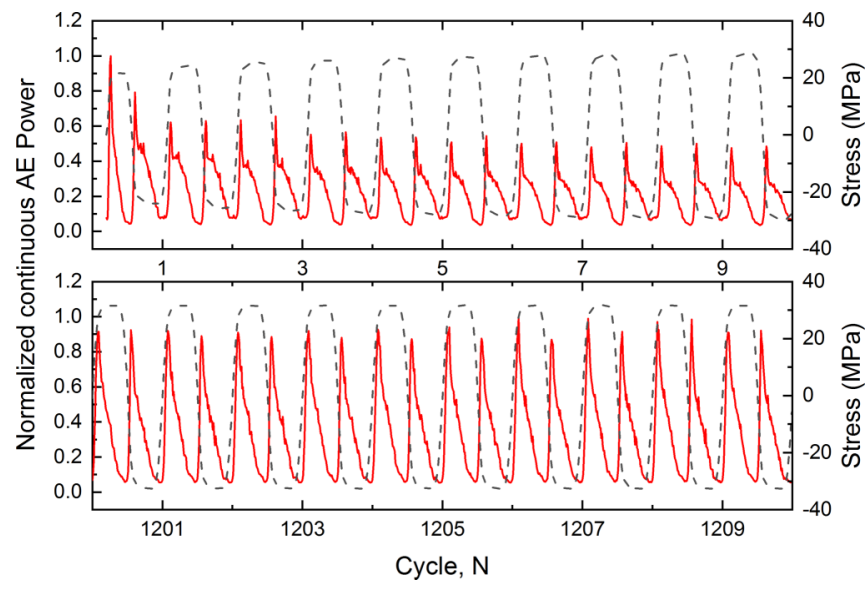

FIG. 5. Evolution of the applied stress (black dashed line) and of the AE power $\frac{d E}{d t}$ (red line) during a cyclic strain-controlled tension-compression $\left(R_{\varepsilon}=\frac{\varepsilon_{\min }}{\varepsilon_{\max }}=-1 ; \Delta \varepsilon=\varepsilon_{\max }-\varepsilon_{\min }=0.95 \%\right)$ test on a nonannealed polycrystalline Al sample. (top) During the initial hardening stage; (bottom) During the secondary hardening stage. 


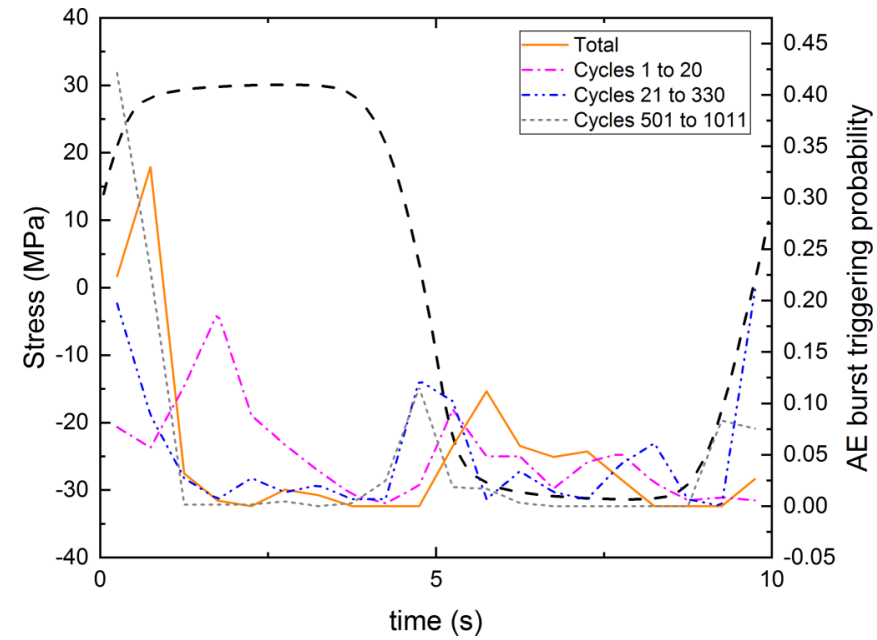

FIG. 6. Occurrence of the discrete AE (burst) activity within the loading cycle, for a test $\left(R_{\varepsilon}=\frac{\varepsilon_{\min }}{\varepsilon_{\operatorname{mix}}}=-1 ; \Delta \varepsilon=\varepsilon_{\max }-\varepsilon_{\min }=\right.$ $0.95 \%$ ) performed on an annealed polycrystalline Al sample. All the bursts recorded during the different stages of deformation have been gathered together to build these histograms.

of the AE wave relates to the surface swept out by all the dislocations during the avalanche, and can be therefore linked to the strain increment $\varepsilon_{\mathrm{p}}$ [23]. We recall that the recorded emitted power, sampled at $100 \mathrm{~Hz}$, encompasses both the mild and wild components of AE, the latter being, however, smoothed by the low sampling frequency. The burst activity was roughly symmetric in tension and compression, concentrated at plastic yield and, to a lesser extent, during the plastic plateau (Fig. 6). No bursts were triggered during unloading phases. This is in agreement with their plastic origin. Some asymmetry (more bursts triggered under tension) possibly emerged during the secondary hardening stage.

Similarly, for all stages of the cyclic deformation analyzed here (i.e., before stage II crack propagation; see above), the AE power was maximal at the yield stress, and symmetric in tension and compression over the loading cycle, in agreement with the assumption about its plastic origin, with the exception of the very first cycle for which a maximal $\mathrm{AE}$ activity was observed when the yield conditions were reached for the first time (under tension in our tests), see Fig. 5.

As we have shown previously, in the case of deformation of micropillars, the wildness measure $W$ can be reliably estimated from an analysis of strain and/or stress increments/decrements on the loading curve [35]. In the present set of experiments, focused on the behavior of bulk samples, even the largest avalanches were undetectable from the macroscopic loading curves given the limited sensitivity and the recording frequency of our stress and strain measurements. We therefore defined an AE proxy of wildness denoted by $W_{A E}$, as the fraction of $\mathrm{AE}$ energy released through detected $\mathrm{AE}$ bursts, once the instrumental noise has been removed. Considering a part of a test (e.g., some consecutive cycles), the wildness $W_{A E}$ is calculated from the ratio between the sum of the corrected (instrumental/environmental noise removed) burst energies detected during this period in one hand, and the total AE power integrated over the same period, and corrected from instrumental noise as well, on the other hand. $W_{A E}$ is a useful dimensionless measure of the plastic intermittency in bulk samples, especially when one can track its evolution through successive cycles.

For monotonic tests (tension or compression) on hcp single crystals (Ice, $\mathrm{Cd}, \mathrm{Zn}$ ), $W_{A E}$ was found to be always larger than 0.95 , actually almost 1 in ice, the paradigm of wildness. By contrast, it was only of a few \% or less in monotonic uniaxial tension of fcc single crystals $(\mathrm{Cu}, \mathrm{Al})$, illustrating the strong effect of crystal symmetry on plastic intermittency [26]. As we describe below, $W_{A E}$ falls to even lower values during the cyclic loading of $\mathrm{Al}$ samples, as the result of progressive dislocation patterning.

Figure 2 summarizes the evolution of the average number of detected bursts per cycle, $\frac{d n}{d N}$, and of the parameter $W_{A E}$, through fatigue life for samples without initial annealing, which are likely to have a relatively large $\rho_{i}$. Overall, both parameters exhibit very low values: generally less than 1 detected burst per cycle, and $W_{A E}<10^{-4}$. This illustrates the extreme mildness of $\mathrm{Al}$ at bulk scales, in agreement with the fact that a wild-to-mild transition takes place in this material for a system size $L \approx 5-10 \mu \mathrm{m}$ [35]. Nevertheless, some episodic discrete AE bursts do persist at those scales, exhibiting a clear evolution through cyclic loading, which follows the dislocation pattern evolution described above $[45,46]$. Both $\frac{d n}{d N}$ and $W_{A E}$ first strongly decrease during the initial hardening stage, by more than two orders of magnitude in the case of the wildness measure. This can be interpreted as a progressive suppression of dislocation avalanches as a cell structure emerges. Gathering all the AE bursts recorded during this initial hardening stage (A), we obtain a power law cumulative distribution of $\mathrm{AE}$ energies, $P(>E) \sim E^{-\tau_{E}+1}$, with the exponent $\tau_{E}=1.70 \pm 0.4$ determined from a robust maximum likelihood methodology [49], the uncertainty being large owing to the limited number of detected bursts (Fig. 7).

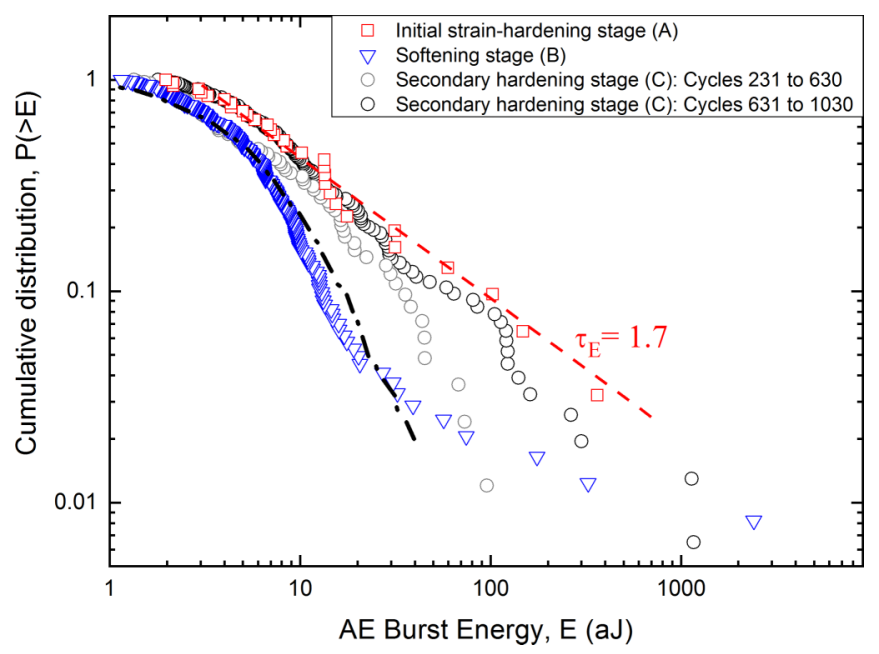

FIG. 7. Cumulative probability distributions for AE burst energies recorded at different stages of cyclic deformation (straincontrolled tension-compression with $R_{\varepsilon}=\frac{\varepsilon_{\min }}{\varepsilon_{\max }}=-1 ; \Delta \varepsilon=\varepsilon_{\max }-$ $\varepsilon_{\min }=0.95 \%$ ) for a test on a nonannealed Al sample. The black dash-dotted line represent a corresponding log-normal distribution for the softening stage, while the red dashed line exemplifies the power law tail associated with the initial strain-hardening stage. 


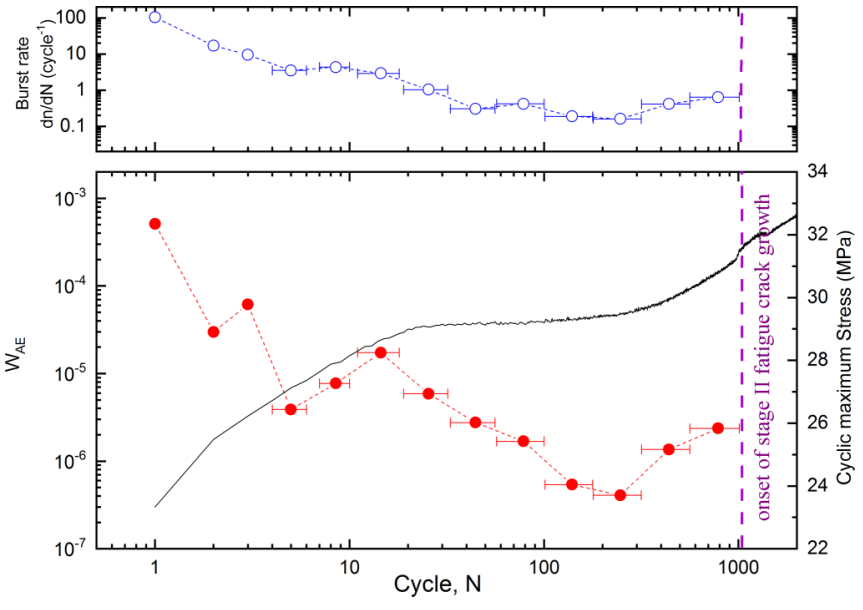

FIG. 8. Same as Fig. 2 for a tension-compression $\left(R_{\varepsilon}=\frac{\varepsilon_{\min }}{\varepsilon_{\max }}=\right.$ $\left.-1 ; \Delta \varepsilon=\varepsilon_{\max }-\varepsilon_{\min }=0.95 \%\right)$ test on an annealed polycrystalline Al sample.

We can conclude that, during this stage, two phenomena compete: collective dislocation dynamics, governed by longranged elastic interactions among dislocations, and the emergence of a self-organizing network controlled by short-range interactions and progressively inhibiting collective effects. If the first process is intrinsically scale-free and associated with power-law distributions of avalanche energies, the second one is characterized by an internal length scale $l$ decreasing as the material hardens, which explains the decrease of the wildness measure in this initial hardening stage.

Previous TEM observations $[45,46]$ showed that the softening stage (B), shown in Fig. 2, is associated with a severe re-organization of the transient initial cell structure. Our results suggest that this reorganization occurs partly through (relatively) large dynamical instabilities, especially at the end of this stage, with a peak of wildness measure (Fig. 2). The distribution of AE energies shows several outliers coexisting with log-normal-like statistics for the events with small sizes (Fig. 7). This distribution presents a clear evidence of the possible coexistence of mild and wild fluctuations in crystal plasticity (see, also, Ref. [26]) and shows on one side a relation between strong dislocation patterning, hardening, and mildness, and on the other side a link between microstructural reorganization, softening, and wildness. All nonannealed samples showed similar behavior of this type.

After this softening stage, we observe that both quantities, $\frac{d n}{d N}$ and $W_{A E}$, reach another minimum before starting to increase slowly during the secondary hardening stage $(\mathrm{C})$ lasting hundreds of cycles and accompanied by the development of a well-defined wall/ladder structure and PSBs (see Sec. III). This evolution brings the distribution of AE energies back towards a power law (Fig. 7). In other words, plastic avalanches do exist during this stage with a power law tail of the energy distribution developing as one approaches the onset of stage II fatigue crack growth. While these observations remind us of the "strain avalanches" discussed by Laird and co-workers, the present data do not allow us to determine whether the AE bursts are signatures of extended microstructural rearrangements in the matrix such as those recorded in the earlier stages of fatigue, or the result of fast and coordinated dislocation motions along PSBs.

We also conducted similar experiments with samples annealed at $250{ }^{\circ} \mathrm{C}$ with the goal of reducing the initial dislocation density $\rho_{i}$. For those samples the softening stage (B) was suppressed (Fig. 8) and the initial hardening was followed by a plateau preceding secondary hardening. This suggests a more progressive evolution towards a well-defined wall pattern, without the need of complete reorganization of an initial, transient cell structure. In these tests both $\frac{d n}{d N}$ and $W_{A E}$ were initially much larger than in nonannealed samples, which is consistent with the understanding that annealed samples have a larger initial mean-free path for dislocations (scaling as $1 / \sqrt{\rho_{i}}$ ). During the first $\sim 200$ cycles, both $\frac{d n}{d N}$ and $W_{A E}$ rapidly fall down to small values comparable to those observed in nonannealed samples. The peak of wildness observed around $N=200$ in nonannealed samples was not recovered here, consistent with the absence of softening stage. This progressive fading of intermittent plasticity under
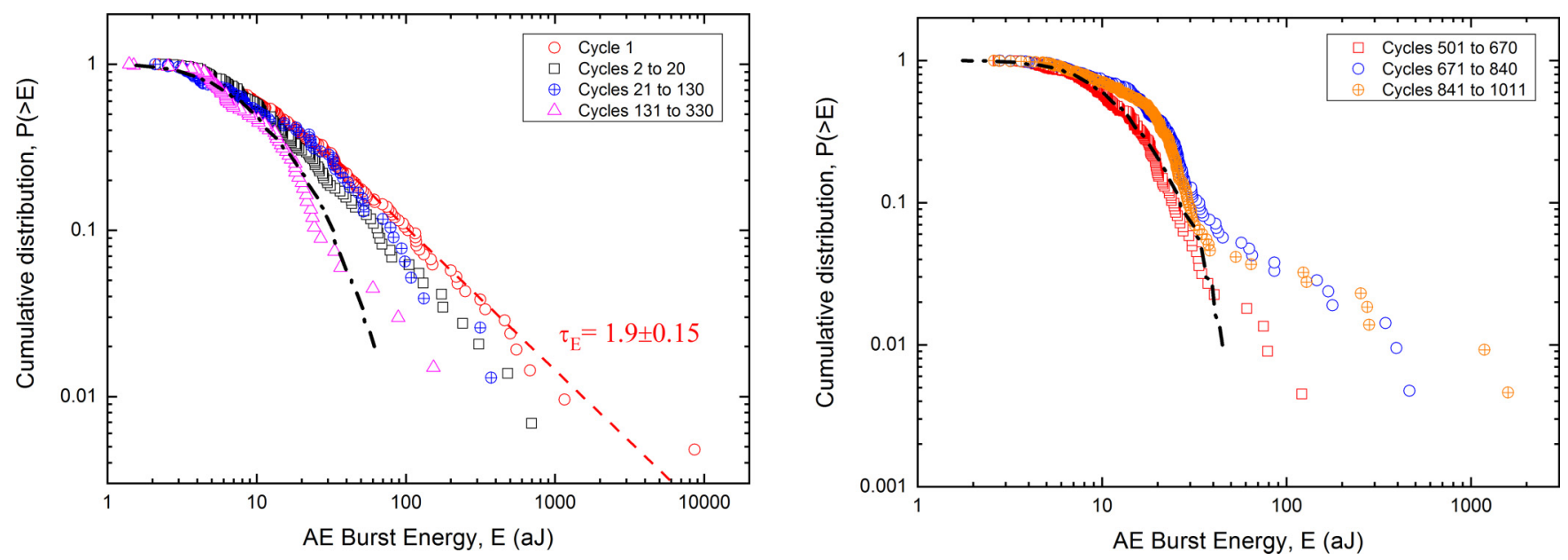

FIG. 9. Same as Fig. 7 for a test on an annealed sample. (a) For the initial stages of cyclic deformation (initial hardening and stress plateau); (b) For the secondary hardening stage. The black dash-dotted lines represent a corresponding log-normal distribution for (a) cycles 131 to 330 , and (b) cycles 501 to 670 . 
cycling is also illustrated by the evolution of the cumulative distribution of $\mathrm{AE}$ burst energies, which drifts away from a power law at the onset of cycling to an essentially log-normal distribution with few outliers reached after few hundreds of cycles [Fig. 9(a)].

Much like for nonannealed samples, both the burst rate and the wildness measure slowly regrow during the secondary hardening stage (Fig. 8), whereas a power-law tail appear to be emerging again in the distribution of $\mathrm{AE}$ energies as the system reaches the onset of stage II fatigue crack growth [Fig. 9(b)].

\section{DISCUSSION AND CONCLUSIONS}

From the results presented above, several conclusions can be drawn:

(1) During the initial stages of cyclic deformation, up to the onset of secondary hardening, a strong link is revealed between dislocation patterning, hardening/softening and the mildness/wildness of collective dislocation dynamics. This can be qualitatively rationalized as follows: with accumulating cyclic deformation, the total dislocation density increases and a dislocation pattern emerges. This leads to strain hardening and the reduction of the internal length scale $l$, which causes, in turn, a decrease of the degree of wildness of the plastic flow. The strong difference in the degree of initial wildness between nonannealed (higher $\rho_{\mathrm{i}}$ ) and annealed samples (lower $\rho_{\mathrm{i}}$ ), as well as the fact that the severe microstructural reorganization (strong variations of $\rho$ ) accompanying softening (when present) is associated with a transient increase of wildness, are also consistent with this conceptual framework. In our previous papers, we studied the sensitivity of the degree of intermittency of plastic flow (wildness) to the crystal structure [26], to the external size $L$, and to the internal scale that was artificially controlled by the manipulation of disorder (alloying) [35]. Here, we (partially) sweep the wild-to-mild transition in pure $\mathrm{Al}$ within a given test by strain-hardening the material during cyclic loading. In this sense, our present study reveals the sensitivity of the degree of intermittency of plastic flow (wildness) to the (emergent) internal length scale $l$.

Mudrock et al. [50] analyzed plastic intermittency on an Al-Mg Alloy loaded monotonically under strain-rate control, and reported power law scaling in the Fourier spectrum of stress fluctuations after plastic yield. This might appear contradictory with what is reported above. Note, however, that the frequency scales explored by these authors with their measurements $(0.01-5 \mathrm{~Hz})$ were orders of magnitude smaller than those explored by AE. It is, therefore, not clear whether fluctuations occurring at frequencies below $5 \mathrm{~Hz}$ could be associated (or not) with dislocation avalanches giving rise to inertial effects and AE waves. This point would be worth exploring in future work.

(2) We observed that the decrease of wildness with cycling is accompanied by an increase of the exponent $\tau_{E}$ (Fig. 10), meaning that a milder plasticity can be associated with larger values of $\tau_{E}$. Although the error bars associated with these data are large, especially for the mixed distributions where the number of power law distributed events is limited, this anticorrelation between $W_{A E}$ and $\tau_{E}$ is consistent with our previous findings and theoretical developments [26,35]. In particular,

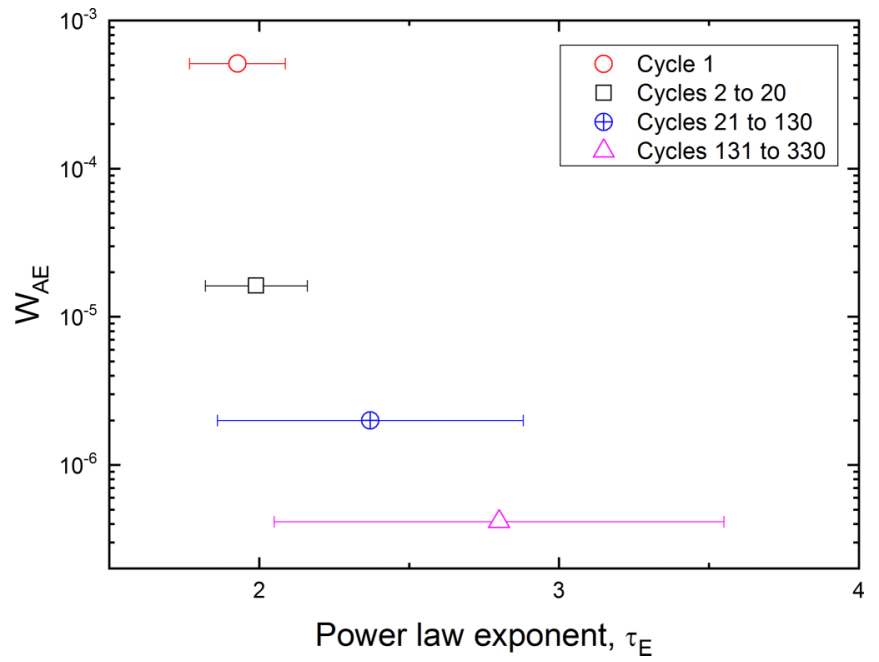

FIG. 10. Relationship between the power law tail exponent of the distributions of $\mathrm{AE}$ burst energies, $\tau_{E}$, and the $\mathrm{AE}$ proxy of wildness, $W_{A E}$ (averaged over the indicated ranges of cycles) for the test shown in Fig. 5, on an annealed polycrystalline Al sample.

such anticorrelation follows from a mean field model for collective dislocation dynamics summarized by the stochastic differential equation, $\frac{d \rho_{m}}{d \gamma}=A-C \rho_{m}+\sqrt{2 D} \rho_{m} \xi(\gamma)$, where $\rho_{m}$ is the spatially averaged mobile dislocation density, $\gamma$ is the average shear strain, $A>0$ is the net nucleation rate, and $C>0$ is the rate of mutual annihilation/immobilization of dislocation pairs. In this simplified framework, long-ranged stochastic interactions are described by a multiplicative noise involving a standard white noise $\xi(\gamma)$ with zero average and $\delta$-type correlations. The noise intensity $D$ quantifies the intensity of fluctuations experienced by a representative volume due to interactions with the rest of the system [26]. The stationary probability distribution for $\rho_{m}$ predicted by this model is characterized by a well-defined scale $\rho_{m}=A /(C+D)$ at small sizes coexisting with a power law tail with exponent $\kappa=$ $1+\frac{C}{D}$ at large sizes, much like in our present observations. The relative importance of the power law tail measured by the wildness parameter $W$, was shown to depend uniquely on $C / D$, thus arguing for a universal $W(\kappa)$ relationship: the larger the $C / D$ dimensionless ratio, the larger the exponent and the smaller the wildness [35]. The universality of this relation was experimentally confirmed based on the detailed analysis of strain fluctuations in compression tests on $\mathrm{Al}$ and $\mathrm{Al}$-alloy micropillars of various sizes, over the entire wild-to-mild transition [35]. This prediction is qualitatively consistent with the present results for $W_{A E}\left(\tau_{E}\right)$ (Fig. 10). We note however that (i) in these experiments on bulk $\mathrm{Al}$ the wildness is always very small, i.e., we are exploring only the very end of the transition, and (ii) AE-deduced $W_{A E}$ is only a proxy of true wildness. All this precludes, at this point, a quantitative comparison between the data and the model predictions. However, if we recall the connection between the noise intensity and the internal length scale, $D \sim l / b$ proposed in Ref. [35], the progressive fading of plastic intermittency with cycling loading in the early stages of fatigue can be interpreted as a decrease of $l$ (so of $D$ ). The concurrent increase of the power law exponent resulting from microstructural evolution 
and dislocation patterning could be then anticipated based on the qualitative reasoning proposed above.

(3) As we mentioned in Sec. III, dislocation patterning under cyclic loading of fcc metals gives rise eventually to PSB formation, which is known to be the phenomenon at the origin of fatigue crack initiation. In pure $\mathrm{Al}$, the emergence of PSBs [Fig. 3(c)] is accompanied by a slow, secondary hardening, as well as by a reincrease of $W_{A E}$ of about one order of magnitude (in both nonannealed and annealed samples). Hence, the unequivocal relationship between mildness and hardening, reported previously [35] and discussed above, breaks down here, suggesting another origin of acoustically detectable avalanches. Instead of microstructural rearrangements within the wall-and-ladder matrix structure, the recorded AE bursts may be the signature of collective and coordinated dislocation motions along PSBs leading to the formation of incipient microcracks. If so, the increase of the burst rate as well as the evolution of the tail of the distribution of AE energies as the system approaches the onset of stage II fatigue is the signature of plastic instabilities associated with microcrack nucleation along primary slip planes, reviving and supporting the initial proposal of Laid and coworkers made more than 30 years ago $[10,12]$. A possible emergent asymmetry of the discrete AE (more bursts triggered under tension) might also be consistent with incipient microcracking, but this point has to be taken with caution owing to limited statistics (Fig. 6).

\section{ACKNOWLEDGMENTS}

This work was partly supported by the French ANR-2008 grant EVOCRIT (Grant No. BLAN08-3_341308). We thank two anonymous referees for interesting comments and suggestions.
[1] V. Esslinger, R. Kieselbach, R. Koller, and B. Weisse, Eng. Failure Anal. 11, 515 (2004).

[2] S. Bhaumik, M. Sujata, and M. Venkataswamy, Eng. Failure Anal. 15, 675 (2008).

[3] W. Lee, L. Nguyen, and G. S. Selvaduray, Microelectron. Reliab. 40, 231 (2000).

[4] R. I. Stephens, A. Fatemi, R. R. Stephens, and H. O. Fuchs, Metal Fatigue in Engineering (John Wiley \& Sons, New York, 2000).

[5] M. D. Sangid, Int. J. Fatigue 57, 58 (2013).

[6] C. Laird, P. Charsley, and H. Mughrabi, Mater. Sci. Eng. 81, 433 (1986).

[7] H. Mughrabi, Metall. Mater. Trans. A 40, 1257 (2009).

[8] H. Mughrabi, R. Wang, K. Differt, and U. Essmann, in Fatigue Mechanisms: Advances in Quantitative Measurement of Physical Damage (ASTM International, Philadelphia, 1983), pp. 5-45.

[9] S. Suresh, Fatigue of Materials, 2nd ed. (Cambridge University Press, Cambridge, UK, 2001).

[10] B.-D. Yan and C. Laird, Acta Metall. 33, 2023 (1985).

[11] L. Buchinger, B. Da Yan, and C. Laird, Mater. Sci. Eng. 70, 161 (1985).

[12] F. Lorenzo and C. Laird, Mater. Sci. Eng. 52, 187 (1982).

[13] S. I. Hong and C. Laird, Mater. Sci. Eng. 89, 63 (1987).

[14] J. Weiss and J. R. Grasso, J. Phys. Chem. B 101, 6113 (1997).

[15] M. C. Miguel, A. Vespignani, S. Zapperi, J. Weiss, and J. R. Grasso, Nature (London) 410, 667 (2001).

[16] M. Zaiser, Adv. Phys. 55, 185 (2006).

[17] S. Papanikolaou, Y. Cui, and N. Ghoniem, Modell. Simul. Mater. Sci. Eng. 26, 013001 (2017).

[18] R. Becker and E. Orowan, Z. Phys. 79, 566 (1932).

[19] R. Tinder and J. Trzil, Acta Metall. 21, 975 (1973).

[20] R. Maass and P. Derlet, Acta Mater. 143, 338 (2018).

[21] T. Richeton, J. Weiss, and F. Louchet, Acta Mater. 53, 4463 (2005).

[22] T. Richeton, P. Dobron, F. Chmelik, J. Weiss, and F. Louchet, Mater. Sci. Eng. A 424, 190 (2006).

[23] J. Weiss, T. Richeton, F. Louchet, F. Chmelik, P. Dobron, D. Entemeyer, M. Lebyodkin, T. Lebedkina, C. Fressengeas, and R. J. McDonald, Phys. Rev. B 76, 224110 (2007).
[24] J. Weiss and D. Marsan, Science 299, 89 (2003).

[25] J. Weiss and M.-C. Miguel, Mater. Sci. Eng. A 387-389, 292 (2004).

[26] J. Weiss, W. Rhouma, T. Richeton, S. Deschanel, F. Louchet, and L. Truskinovsky, Phys. Rev. Lett. 114, 105504 (2015).

[27] R. Madec, B. Devincre, and L. P. Kubin, Phys. Rev. Lett. 89, 255508 (2002).

[28] R. Madec, B. Devincre, L. Kubin, T. Hoc, and D. Rodney, Science 301, 1879 (2003).

[29] B. Devincre, T. Hoc, and L. Kubin, Science 320, 1745 (2008).

[30] D. Rouby, P. Fleischman, and C. Duvergier, Philos. Mag. A 47, 671 (1983).

[31] A. Slimani, P. Fleischmann, and R. Fougères, J. Phys. III France 2, 933 (1992).

[32] C. Fressengeas, A. J. Beaudoin, D. Entemeyer, T. Lebedkina, M. Lebyodkin, and V. Taupin, Phys. Rev. B 79, 014108 (2009).

[33] M. Montagnat, J. Weiss, P. Duval, H. Brunjail, P. Bastie, and J. Gil Sevillano, Philos. Mag. A 86, 4259 (2006).

[34] B. Jakobsen, H. F. Poulsen, U. Lienert, J. Almer, S. D. Shastri, H. O. Sorensen, C. Gundlach, and W. Pantleon, Science 312, 889 (2006).

[35] P. Zhang, O. G. Salman, J. Y. Zhang, G. Liu, J. Weiss, L. Truskinovsky, and J. Sun, Acta Mater. 128, 351 (2017).

[36] D. M. Dimiduk, C. Woodward, R. LeSar, and M. D. Uchic, Science 312, 1188 (2006).

[37] S. Brinckmann, J. Y. Kim, and J. R. Greer, Phys. Rev. Lett. 100, 155502 (2008).

[38] M. Sauzay and L. P. Kubin, Prog. Mater. Sci. 56, 725 (2011).

[39] M. Zaiser and S. Sandfeld, Modell. Simul. Mater. Sci. Eng. 22, 065012 (2014).

[40] L. Kubin, Dislocations, Mesoscale Simulations and Plastic Flow (Oxford University Press, Oxford, 2013).

[41] M. Staker and D. Holt, Acta Metallurgica 20, 569 (1972).

[42] P. Hahner, K. Bay, and M. Zaiser, Phys. Rev. Lett. 81, 2470 (1998).

[43] L. Kubin and M. Sauzay, Acta Mater. 104, 295 (2016).

[44] S. Deschanel, W. Ben Rhouma, and J. Weiss, Sci. Rep. 7, 13680 (2017). 
[45] M. Videm and N. Ryum, Mater. Sci. Eng. A-Struct. Mater. Prop. Microstruct. Process. 219, 1 (1996).

[46] M. Videm and N. Ryum, Mater. Sci. Eng. A-Struct. Mater. Prop. Microstruct. Process. 219, 11 (1996).

[47] J. Chicois, R. Fougeres, G. Guichon, A. Hamel, and A. Vincent, Acta Metall. 34, 2157 (1986).
[48] N. Kiesewetter and P. Schiller, Phys. Stat. Sol. 38, 569 (1976).

[49] A. Clauset, C. R. Shalizi, and M. E. J. Newman, SIAM Rev. 51, 661 (2009).

[50] R. N. Mudrock, M. A. Lebyodkin, P. Kurath, A. J. Beaudoin, and T. A. Lebedkina, Scr. Mater. 65, 1093 (2011). 